\title{
Erratum to: E-Life: Web-Enabled Convergence of Commerce, Work, and Social Life
}

\author{
Vijayan Sugumaran ${ }^{1(\bowtie)}$, Victoria Yoon $^{2}$, and Michael J. Shaw ${ }^{3}$ \\ 1 Department of Decision and Information Sciences, Oakland University, \\ Rochester, MI, USA \\ sugumara@oakland.edu \\ 2 Virginia Commonwealth University, Richmond, VA, USA \\ ${ }^{3}$ Beckman Institute for Advanced Science and Technology, University of \\ Illinois at Urbana-Champaign, Urbana, IL, USA
}

\section{Erratum to: \\ V. Sugumaran et al. (Eds.): \\ E-Life: Web-Enabled Convergence of Commerce, Work, and Social Life, LNBIP 258, https://doi.org/10.1007/978-3-319-45408-5}

In the original publication of this book the conference numbering in the subtitle was incorrect. It was the 14th Workshop but the subtitle erroneously stated " 15 th Workshop". This has now been corrected. 